\title{
Research on Multiple Spatial Data Gathering Mechanism Based on Geo-information Browser ${ }^{1}$
}

\author{
Ken Chen ${ }^{1,3}$, a Fang Wang ${ }^{2}$, Fang Miao ${ }^{1,3, b}$, Fu-chao Cheng ${ }^{1,3}$ \\ ${ }^{1}$ State Key Laboratory of Geohazard Prevention and Geoenvironment Protection, Chengdu, \\ 610059, China; \\ ${ }^{2}$ College of Computer Science and Technology, Southwest University for Nationalities, \\ Chengdu, 610041, China \\ ${ }^{3}$ Spatial Information Technology Institute, Chengdu 610059, China \\ ack@cdut.edu.cn, ${ }^{a}$ chenken@foxmail.com, ${ }^{a}$ chenken@cdut.edu.cn, b mf@cdut.edu.cn
}

Keywords: Geo-information browser, cloud, G/S model, Information-gathering, HGML

\begin{abstract}
The spatial data presented several characteristics of mass, multiple, isomerism and multiple tenses, its organization and management mechanism is an important direction of research for Digital Earth. The management of grave emergency with regards to a series of spatial and non-spatial data concerning gathering and handling, having put a higher demand forward the ability of information gathering mechanism on client.
\end{abstract}

The current existing client access mechanism such as C/S model lacks of unified data exchange standards, similarly, B/S model cannot handle the spatial data effectively. It is also difficulty to display for complex and massive spatial data in visualized and real-time. That efficiency depends entirely on the network environment and performance of storage equipment. In order to realize the massive spatial data unified dispatching and efficient sharing based on the principle of Information-gathering and Service-polymerization. We put forward a concept of Spatial-data-cloud which based on G/S model, supported by HGML as the standard and criterion of spatial data exchange, presentation, organization, storage and management. It could also be set up a new work mechanism which use Geo-information browser polymerization multiple and massive complex spatial and non-spatial data.

This will provide us a lightweight client called Geo-information browser with which is by the principle Information-gathering and Service-polymerization. It provides emergency management for technical supports such as intelligent decision support, comprehensive research and judgment, and rapid disposal etc. It is the development of basic research of a novel model of Digital Earth.

\section{Introduction}

Because of the emergency such as natural disasters and social problems are mixed and coexist, brings about immeasurable loss to the nation and people's intertwined lives, also restricts the national economy development with comprehensively, coordinately and sustainably. Real-time and effective to handle emergency problems facing the severe situation and challenge, a powerful lightweight Geo-information browser client is indispensability. It is based on the functions such as provide data for users to browse, search, analysis, interoperability, and other functions[1]. Also events for natural disasters could be provided functions such as scientific, intuitive, real-time, fast, smart aided decision, 3D display, integrated judge, rapid disposal and others which could satisfy the popular applications at the same time[2]. This research could polymerization large-scale complex spatial information services to meet the Geo-information browser's increasingly requirements of

${ }^{1}$ Financial Support: (Grant No. NSFC 61071121) "Research on the client polymerization of spatial information network service model.” (No. 11NZYTD09) Fundamental Research Funds for the Central Universities, Southwest University for Nationalities 
spatial information service application. It has important and effective significance to realize spatial information service polymerization.

\section{Research on Geo-information browser service mechanism}

The current existing client access mechanism such as C/S model lacks of unified data exchange standards, similarly, B/S model cannot handle the spatial data effectively. It is also difficulty to display for complex and massive spatial data in visualized and real-time. In view of the above questions, the group did a lot of research and hard work will on popularization application on spatial information. It presents Geo-information browser with a structure of heterogeneous spatial data analysis of multivariate characteristics based on G/S model, which take HGML as the core of exchange sharing platform, polymerization large-scale complex of spatial information services. It makes the services can satisfy the emergency command demands as well as popular services.

Figure 1: Service mechanism framework of Geo-information browser

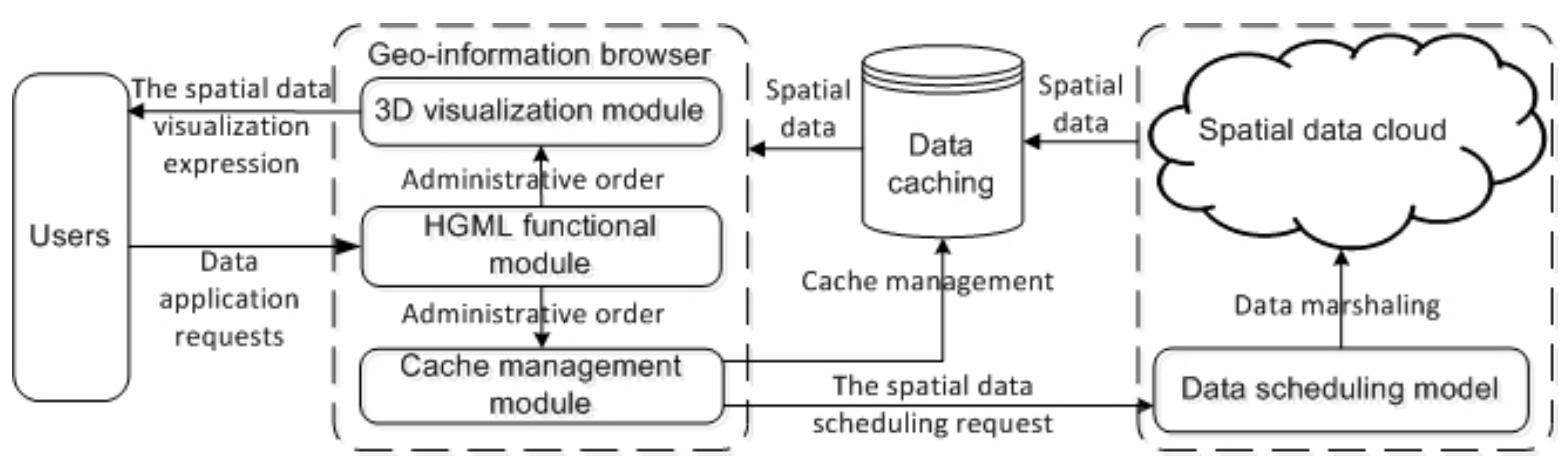

\section{Research plan based on Geo-information browser}

Firstly, starting with the distributed and massive spatial data analysis, study its characteristics in the network services, especially the geographical position relevance. Based on this study to design a work mechanism of a HGML as the core of the spatial data exchange sharing platform, which can apply in geological browser memory management mechanism, memory mapping mechanism, cache management mechanism, and collaboratively research geological browser client polymerization large-scale space information network service. In addition, in a variety of study on the spatial data formats on the basis of a set of HGML to the data format of the data exchange mechanisms, to extend the system to different data format compatibility. Design a study on this issue last working mechanism of the prototype system, give full consideration to disaster in real time and efficient emergency response system needs, and verify the feasibility of design theory in a practical application.

Caching mechanism research. In according to establish a Geo-information browser cache mechanism, so that realizes the efficient polymerization in the client. The major steps are: (1) Study on characteristics of spatial data location, analyze the application on geographical characteristics in the relevance and specificity of spatial data, and provide algorithm model design based on designing and researching the cache mechanism. (2) Design a kind of memory replacement algorithm based on geographic location and a storage mapping mechanism relevant geographic location information, do the client with low system resource consumption to realize effective management and efficient use of the application of the local cache. (3) Research and design a high performance geographic predict model to achieve data smart cache technology. The three sections above, coordinating with the described in the following sections apply to large and complex spatial information services aggregate data scheduling mechanisms, intelligent and efficient implementation of Geo-information browser requirements.

Research on Distributed and massive spatial information data scheduling model. Design data scheduling mechanisms for spatial information in the server farm, the main methods are: (1) 
Design and perfect a set of spatial information characteristic model to accurately express distribution large-scale complex spatial data characteristics. (2) According to the characteristics of spatial information model, design a data scheduling algorithm, which can fast and accurately position and response to data source position. (3) In order to regulate data exchange standards between client and servers, define a distributed data scheduling exchange agreement standard. From the above three sections can establish a kind of spatial information network service which could satisfies standardization and effective in the data scheduling mechanism.

Heterogeneous spatial data exchange mechanism. Design the data exchange mechanism supports a variety of spatial data standards, mainly includes the following two steps: (1) Research existing data formats standard of several common spatial information, analyzes its semantic mapping relationship and the performance difference. (2) From HGML-oriented data format to design a set of high-precision, high efficiency, wide support data exchange mechanism of spatial information data in multiple formats, and has good scalability and full transparency to users. The above two parts in order to achieve broad compatibility of Geo-information browser for a variety of data formats, providing a common platform to address the issue of the emergency data from multiple heterogeneous data is unable to efficiently share.

Research on spatial data and 3d visualization contracted and merged algorithm. For existing research and optimal design of 3D graphics algorithms, combined with the geographical characteristics of spatial information and related characteristics of the network services model to design a set of effective lightweight client. Fully take advantage of the GPU's strong graphics capabilities to achieve the 3D visualization displays of the geospatial information, display capabilities across platforms, interoperability perfect the application of spatial data for users, and improve the efficiency of client handles emergency events and the extent of popular applications.

\section{Conclusion}

In order to solve heterogeneous spactial data sharing liquor and research on HGML as a core, Geo-information browser extensive supports multiple spatial data formats as well as caching mechanism with high performance and provides users with multiple spatial information aggregation services. So as to realize the distributed massive spatial data sharing unified dispatching and efficient coordination. Research a set of spatial information server group applied to the efficient data scheduling model based on G/S model. Distributed massive spatial data in the client polymerization, meanwhile the characteristics of high efficiency standard unified. Proposed performance optimization algorithm based on geographic location performance optimization model and in view of the geographic information 3D visualization display. For Geo-information browser polymerization large-scale complex spatial information services of intelligent and real-time 3D virtualization study provides new ideas. And supports for new generation digital earth is a powerful platform application: (1) Establish a geographically based mass predict model of spatial information data scheduling model based on G/S model. (2) Create a HGML as the core with wide support space information data to the data exchange and standard share platforms. (3) Put forward a kind of lightweight client that can realize massive spatial data real-time $3 \mathrm{D}$ visualization expression, adaptive expression and fast modeling.

\section{Acknowledgments}

The authors would like to acknowledge the Spatial Information Technology Institute the members of, the State Key Laboratory of Geohazard Prevention and Geoenvironment Protection as well as College of Computer Science and Technology Southwest University for Nationalities, owing to their help to produce these examples and ideas presented here and the financial support (Grant No. NSFC 61071121) provided by the National Nature Science Foundation of China, and the Fundamental Research Funds for the Central Universities, Southwest University for Nationalities (No. 11NZYTD09). 


\section{References}

[1] Guo Xirong, Miao Fang, Wang Huajun. Spatial Information G/S network access mode structure were discussed. Computer Applications and Software, 2009(10).

[2] Deng Hongyan, Wu Fang, Wang Zhuoning, Liu Weiwei. Based on the spatial information services Web Service, Computer Engineering and Design [J], 2006 27(23), 4450-4452.

[3] Guo Xirong, Miao Fang, Wang Huajun, Du Genyuan: Initial Discussion on the Architecture of a New Spatial Information Network Service Mode Based on the Digital Earth, ESIAT2009, Published: IEEE Computer Society CPS, 2009, V3, p406-410.

[4] Li Kangrong, Miao Fang: The analysis of multi-dimension spatial data subdivision and storage, ESIAT2009, Published: IEEE Computer Society CPS, 2009, V3, p385-388.

[5] Chengming Ye, Fang Miao, Xiangsheng Kong, Xiaojia Bi, Rui Liu: The oil and gas engineering techniques based on Digital Earth Platform, Proc. SPIE 6795, 679579-1-5(2007).

Figure 2: Caching mechanism structure diagram of Geo-information browser

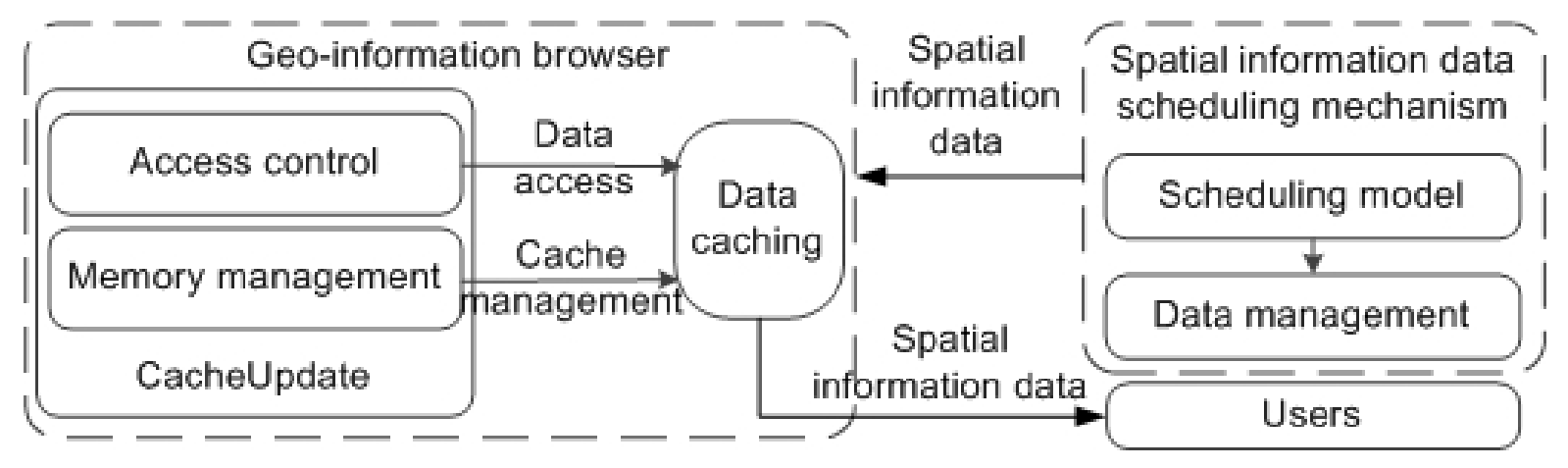

